\title{
Recognition of mango leaf disease using convolutional neural network models: a transfer learning approach
}

\author{
Aditya Rajbongshi', Thaharim Khan ${ }^{2}$, Md. Mahbubur Rahman ${ }^{3}$ Anik Pramanik ${ }^{4}$, Shah Md Tanvir \\ Siddiquee $^{5}$, Narayan Ranjan Chakraborty ${ }^{6}$ \\ ${ }^{1}$ Department of Computer Science and Engineering, Jahangirnagar University, Dhaka, Bangladesh \\ ${ }^{2,4,5,6}$ Department of Computer Science and Engineering, Daffodil International University, Dhaka, Bangladesh \\ ${ }^{3}$ Crowd Realty, Tokyo, Japan
}

\section{Article Info}

Article history:

Received Apr 6, 2021

Revised Jul 18, 2021

Accepted Aug 4, 2021

\section{Keywords:}

Classification

DenseNet201

Mango leaf

Neural network

\begin{abstract}
The acknowledgment of plant diseases assumes an indispensable part in taking infectious prevention measures to improve the quality and amount of harvest yield. Mechanization of plant diseases is a lot advantageous as it decreases the checking work in an enormous cultivated area where mango is planted to a huge extend. Leaves being the food hotspot for plants, the early and precise recognition of leaf diseases is significant. This work focused on grouping and distinguishing the diseases of mango leaves through the process of CNN. DenseNet201, InceptionResNetV2, InceptionV3, ResNet50, ResNet152V2, and Xception all these models of CNN with transfer learning techniques are used here for getting better accuracy from the targeted data set. Image acquisition, image segmentation, and features extraction are the steps involved in disease detection. Different kinds of leaf diseases which are considered as the class for this work such as anthracnose, gall machi, powdery mildew, red rust are used in the dataset consisting of 1500 images of diseased and also healthy mango leaves image data another class is also added in the dataset. We have also evaluated the overall performance matrices and found that the DenseNet201 outperforms by obtaining the highest accuracy as $98.00 \%$ than other models.
\end{abstract}

This is an open access article under the CC BY-SA license.

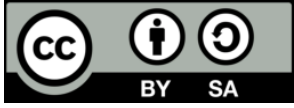

\section{Corresponding Author:}

Aditya Rajbongshi

Department of Computer Science and Engineering

Jahangirnagar University

Dhaka, Bangladesh

Email: adityaraj.jucse@gmail.com

\section{INTRODUCTION}

Mango is one of the tasty and most significant natural product crops which is developed in horticulture. It is sent out to numerous nations as crude or ready foods grown from the ground as handled consumables like ready mango cuts or squeeze crude mango pickle, and so forth mango is plentiful in nutrient $\mathrm{A}$ and $\mathrm{C}$. Disease in leaf makes the photosynthesis be impeded and affect the accrual of the plant.

Unbolting of disease can be completed by farmers by incessant checking of the plant leaves. For littlescope ranchers, early recognizable proof of illness is a lot of conceivable and ready to control the bugs by natural pesticides or by the utilization of negligible measure of compound pesticides. For huge scope ranchers regular observing and early distinguishing proof of illness is unimaginable and it brings about a serious episode of the sickness and irritation development which can't be constrained by natural methods. In this circumstance, farmers are compelled to utilize the noxious synthetic substances to terminate the disease to hold the harvest yield. This issue can be tackled via mechanizing the checking cycle by the utilization of cutting-edge picture handling 
methods. The proposed work aims are making a model involved in disease detection which is performed by using the technique of convolutional neural network $(\mathrm{CNN})$ which is a part of artificial neural network (ANN).

Very few research has been performed regarding the recognition of mango leaf disease. But there have some limitations and most of the approaches were used traditional machine learning algorithms.

Bai et al. [1] present a technique for bird acknowledgment based on Inception-v3. The objective of the LifeCLEF2019 Bird Recognition is to identify and arrange 659 fledgling species inside the gave soundscape chronicles. Log-Mel spectrograms are removed as highlights and Inception-v3 is utilized for bird sound recognition. Dhankhar [2] proposed a method for the use of feature extraction of facial expressions with a blend of neural organization for the acknowledgment of various facial feelings. A human mind can have a parcel of feelings yet this paper manages the fundamental 7 feelings. This paper manages the exceptionally effective model of the joined models of VGG 16 and ResNet50 expanding the productivity to 92.4\%. C. Lin et al. [3] focuses on chromosome classification through a deep learning model. Although the deep learning-based Inception engineering has yielded cutting-edge execution. In this way, they build up a programmed chromosome grouping which is called CIR-Net dependent on Inception-ResNet. Lin et al. [4] proposed a transfer learning-based strategy is presented for traffic sign acknowledgment. It also decreases the measure of preparing information as well as mitigates calculation cost utilizing the Inception-v3 model. The outcomes accomplish in this work are up to $99.18 \%$ of acknowledgment exactness at 0.05 learning rate (normal precision of $99.09 \%$. Ferreira et al. [5] defined a model for the characterization of breast cancer histology pictures.

The pre-owned organization of this proposed work is to perform the characterization with InceptionResnet V2. To defeat the absence of information, information enlargement is performed as well. Rahman et al. [6] proposed another work which is to recognize different local birds of Bangladesh from image information. Here mainly four methodologies to be specific Inception v3 without transfer learning, Inceptionv3 with transfer learning, MobileNet without transfer learning, and MobileNet with transfer learning for figuring out how to achieve the errand. Mukti et al. [7] introduced a Transfer Learning-based CNN model that was created for the distinguishing proof of plant disease exactly. The dataset, they have utilized is comprised of 70295 preparing pictures and 17572 approval images holding 38 distinct classes of plant leaves pictures. They have focused predominantly on the ResNet50 network, a well-known CNN model as a preprepared model in Transfer Learning. Sharmin et al. [8] proposed fish determination in freshwater.

Another more approach is also included herewith as setting up 4 types of features then grayscale image created from the color image. Again through this histogram model which is appeared during the time of conversion performed segmentation. For recognizing the fish 3 classifiers are used in this model among them SVM performs well. Prakash et al. [9] proposed another work on various infections which is responsible for the influence of mango natural product profitability. This paper additionally represents precaution ways and solutions for infection. The framework demonstrates in this paper have the capacity to identify leaf-based diseases however there are many significant infections that influence the efficiency of the mango crop. Shukla et al. [10] proposed one work on Image processing which is one of the fundamental strides for holding innovation in a picture that might be debased because of a few commotions or because of different causes. This paper is an essence of a few Image Preprocessing procedures that can be utilized. Lei et al. [11] proposed one work on Segmentation of images which is significant for picking appropriate territory of interest in a picture. This is critical to lessen handling time that might be needed to deal with rather whole picture. This paper shows a new segmentation strategy called fast and robust fuzzy c-means calculation. The magnificence of this calculation is that it consequently picks a bunch that contains illness. Likewise, it negates indicating preferences over customary FCM calculation. Patel et al. [12] proposed one work that emphasizes every feature of the image to be separated. Highlights that can be extricated from the picture may be of different sorts like worldwide or nearby. In that, there are subcategories like surface-based or colorbased features. Haralick et al. [13] proposed one work that portrays some effectively process-able textural features dependent on gray tone spatial conditions and shows their application in category-identification undertakings of three various types of picture information, two sorts of choice guidelines. These outcomes demonstrate that the effective process able textural includes likely have overall appropriateness for a wide assortment of picture grouping applications. Hu et al. [14] proposed another method that is a feature in MATLAB-based framework is by utilizing gray level co-event matrix (GLCM). MATLAB has an infabricated capacity for drawing GLCM. This paper gives conditions to computing different surface-based highlights from the GLCM grid. Padmaja et al. [15] proposed one method which is on feature extraction, for effectual diseases discovery it is important to make a legitimate determination of highlights that best depicts the given sickness is significant. This paper delineates various highlights determination calculations and highlights scoring techniques.

The main focus of this work is to recognize the diseases of mango leaves with some prominent models of CNN. There are four types of diseases that are used for recognition which are also defined as 
classes into the model along with the set of healthy leaves. DenseNet201, InceptionResNetV2, InceptionV3, ResNet50, ResNet152V2, Xception all these models are used here and then our result is weighed on the principle of seven performance matrices such as accuracy, F1 score, precision, sensitivity, specificity, FNR, FPR. The concise tracing of our study is given below, which narrates the whole study precisely:

- Firstly create an automated method for recognizing the disease of mango leaves.

- This proposed study gives better accuracy for making a depth observation among the various model of CNN.

- The accuracy of this proposed system is good enough than other methods because of the implementation of transfer learning among all the classes.

- This proposed work is debunked in such an effective way which make it competitive than other demonstrated model for mango leaves diseases detection.

\section{RESEARCH METHOD}

In this part, the proposed work is described in a full manner like data preprocessing, description of model, training, and testing of the data set, classifications, and also the description of the dataset. Below Figure 1 illustrate the procedure of the proposed work.

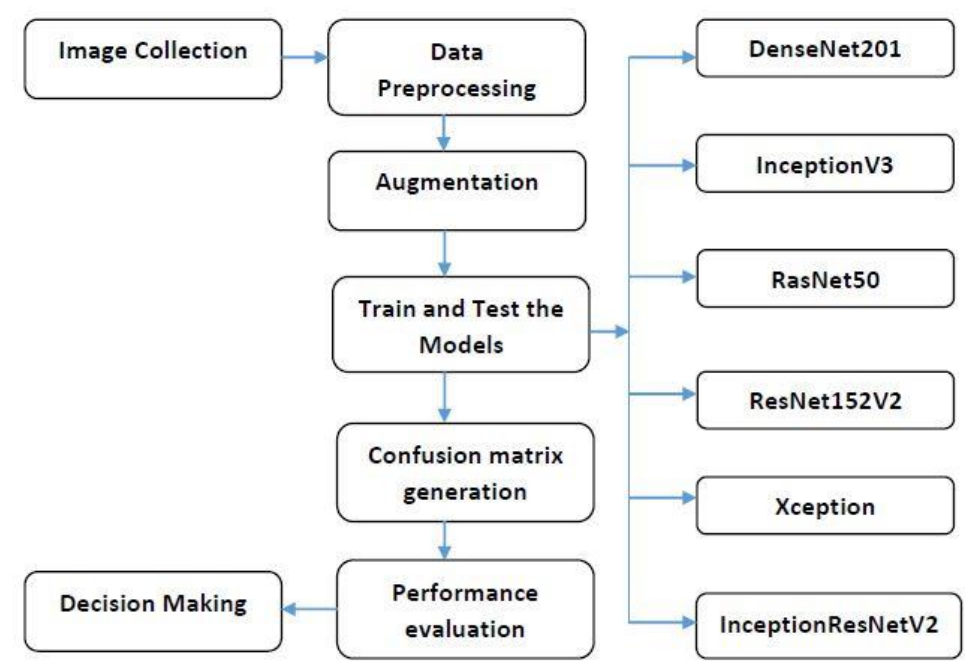

Figure 1. Implementation procedure of the proposed work

\subsection{Dataset acquisition}

To develop the first dataset, the whole mango leaf was shoot. Around 1000 pictures were assumed control over a fourteen-day. Pictures of diseases were taken using a couple of genotypes of mango to furnish the profound learning model with the total range of indications for every illness. The pictures were then metamorphosed by the means of data augmentation to make the auxiliary dataset. These datasets were screened to target model efficiency with whole leaf images, yet a few images contrasted with more edited leaves. The central speculation was that the pictures of edited leaves would upgrade model efficiency to appropriately perceive disease as the informational collection was more prominent. Here Figure 2 shows some data set from 5 different classes of the dataset.
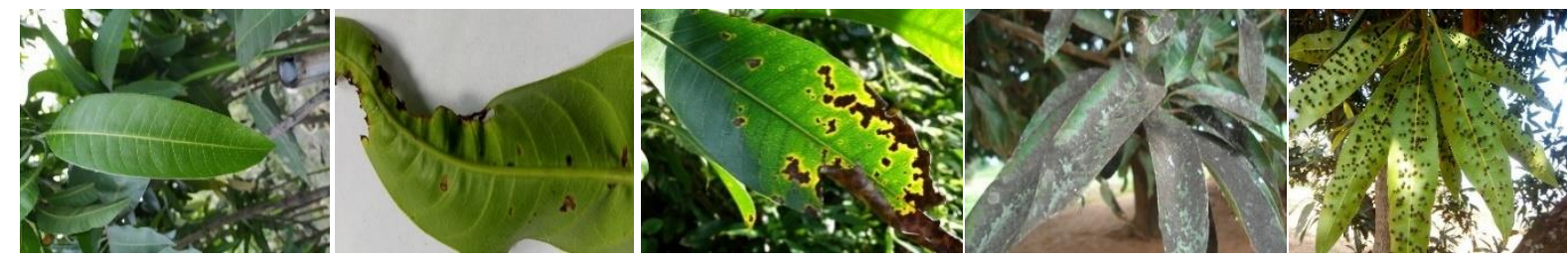

Figure 2. Visualization of dataset (a) Healthy leaf (b) Powdery mildew (c) Anthracnose (d) Red rust (e) Gall machi 


\subsection{Data preprocessing}

Data preprocessing refers to the transformations of the raw data before the dataset is trying to process with any other algorithm. When the raw dataset is trying to process with any other algorithm in CNN then this will lead to a bad classification result [16]. Data augmentation is performed in this regard for reducing the bad classification problem. Diversity of training data and to increase the amount of data Data augmentation [17] is used. Class imbalance problems in the data set can also be solved to some extent with data augmentation. After performing the augmentation there are almost 500 images for each target class which increases the amount of data set to a huge extend. After the augmentation, the data set is ready to split for training and testing purposes. We use the ratio of $80 \%-20 \%$ for train and test operation in the dataset.

\subsection{Model description}

The first layer of a CNN model is convolutional layer which is consider as an input layer. The output of CNN can be denoted mathematically as follows:

$$
C_{y}^{n}=\mathrm{f}\left(\sum_{\mathrm{x} \in \mathrm{My}} p_{x}^{n-1} * q_{x y}^{n}+r_{y}^{n}\right)
$$

Where, $C_{y}$ represents the set of output feature maps, $M_{y}$ represents the set of input maps, $q_{x y}$ represents the kernel for convolution, $r_{y}$ represents the bias term. Below Figure 3 depicts the working procedure for $\mathrm{CNN}$ in this proposed work.

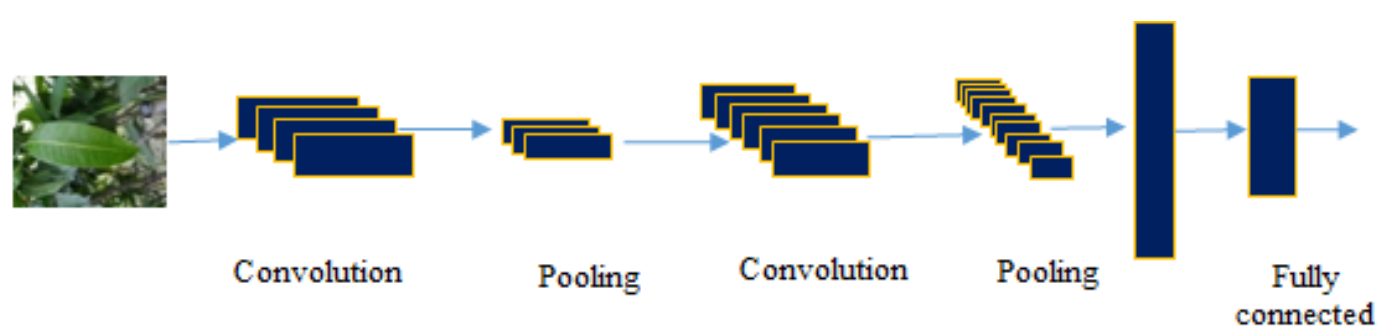

Figure 3. Working procedure of CNN in mango disease detection

Again for the batch normalization, the layer is usually performed between the convolutional layer and the ReLU layer. ReLU layer is an activation layer that is mainly used for adding some non-linearity into the network by changing all the negative activation values to 0. It speeds up furthermore, diminishes the sensitivity of the model. In this layer, the activation of each layer is standardized by subtracting the smaller than mini-batch means and separating by the mini-batch standard deviation. This is trailed by moving the input by an offset $\beta$ and afterward scaling it by a factor $\gamma$.

The mathematic representation of the batch normalization output is given below,

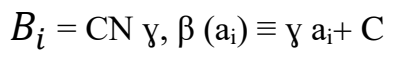

$a_{i}$ is the normalization of activation which is given by the equation,

$$
\mathrm{a}_{\mathrm{i}}=\frac{a_{i+\mu B}}{\sqrt{\sigma^{2} B+\epsilon}}
$$

$\mu B$ is the mini batch mean $\sigma^{2} B$ is mini batch variance

Again there always a softmax layer is present with output layer. Softmax layer always produce output based on probability. The mathematical equation of softmax is given below,

$$
\mathrm{P}\left(\mathrm{C}_{\mathrm{x}} \mid \mathrm{m}, \theta\right)=\frac{\mathrm{P}(\mathrm{m}, \theta \mid \mathrm{Cx}) \mathrm{P}(\mathrm{Cx})}{\sum_{y=1}^{k} \mathrm{P}(\mathrm{m}, \theta \mid \mathrm{Cy}) \mathrm{P}(\mathrm{Cy})}
$$

Except for this traditional way of CNN this proposed work is constructed on the basis of 6 wellknown models for the image data set. DenseNet201, InceptionResNetV2, InceptionV3, ResNet50, ResNet152V2, Xception all these model are used here in this work. For training purposes, we used an output layer of 1000 neurons. Depending on the class we have also added the output layer of 5 neurons as we have only 5 classes into our dataset [18]. 


\subsection{Training and testing}

For training purposes, we are trying to train our data based on 100 epochs as we are trying that 100 times those learning Algorithms will work through the entire training data set. After training the term of testing is coming for getting the accuracy. In this proposed work we are trying to test the newly trained model with the test data set. After testing the images data, $5 \times 5$ confusion matrices are formed for each model. Then we have converted the $5 \times 5$ in binary format which is presented in Table 1 .

Table 1. Confusion matrices as binary format

\begin{tabular}{|c|c|c|c|c|c|}
\hline Method & Class & $\mathrm{TP}$ & FP & $\mathrm{FN}$ & $\mathrm{TN}$ \\
\hline \multirow{6}{*}{ DenseNet201 } & Anthracnose & 59 & 1 & 1 & 239 \\
\hline & Gall Machi & 58 & 1 & 2 & 239 \\
\hline & Healthy Leaf & 59 & 2 & 1 & 238 \\
\hline & Powdery Mildew & 59 & 1 & 1 & 239 \\
\hline & Red Rust & 59 & 1 & 1 & 239 \\
\hline & Anthracnose & 56 & 1 & 4 & 239 \\
\hline \multirow{4}{*}{ InceptionV3 } & Gall Machi & 57 & 2 & 3 & 238 \\
\hline & Healthy Leaf & 59 & 3 & 1 & 237 \\
\hline & Powdery Mildew & 59 & 4 & 1 & 237 \\
\hline & Red Rust & 59 & 1 & 1 & 239 \\
\hline \multirow{5}{*}{ ResNet50 } & Anthracnose & 59 & 1 & 1 & 239 \\
\hline & Gall Machi & 56 & 1 & 2 & 239 \\
\hline & Healthy Leaf & 59 & 5 & 1 & 235 \\
\hline & Powdery Mildew & 58 & 1 & 2 & 239 \\
\hline & Red Rust & 59 & 1 & 1 & 239 \\
\hline \multirow{4}{*}{ ResNet152V2 } & Anthracnose & 57 & 2 & 3 & 238 \\
\hline & Gall Machi & 54 & 2 & 6 & 238 \\
\hline & Healthy Leaf & 58 & 3 & 2 & 237 \\
\hline & Powdery Mildew & 59 & 8 & 1 & 232 \\
\hline \multirow{6}{*}{ Xception } & Red Rust & 56 & 1 & 4 & 239 \\
\hline & Anthracnose & 59 & 1 & 1 & 239 \\
\hline & Gall Machi & 57 & 1 & 3 & 239 \\
\hline & Healthy Leaf & 59 & 3 & 1 & 237 \\
\hline & Powdery Mildew & 59 & 1 & 1 & 239 \\
\hline & Red Rust & 59 & 1 & 1 & 239 \\
\hline \multirow{5}{*}{ InceptionResNetV2 } & Anthracnose & 58 & 1 & 2 & 239 \\
\hline & Gall Machi & 56 & 2 & 4 & 238 \\
\hline & Healthy Leaf & 58 & 4 & 2 & 236 \\
\hline & Powdery Mildew & 59 & 2 & 1 & 238 \\
\hline & Red Rust & 59 & 1 & 1 & 239 \\
\hline
\end{tabular}

\section{Results and Discussion}

For experimental analysis of each model, we have utilized seven performance matrices. The following equations are used to evaluate the seven performance matrices such as accuracy [19], Precision [20], F1 score [21], sensitivity [22], specificity [23], FNR [24], FPR [25].

$$
\begin{aligned}
& \text { Accuracy }=\left(\frac{T P+T N}{T P+F P+F N+T N}\right) \times 100 \% \\
& \text { Precision }=\left(\frac{T P}{T P+F P}\right) \times 100 \% \\
& \text { Sensitivity }=\left(\frac{T P}{T P+F N}\right) \times 100 \% \\
& \text { F1-Score }=\left(\frac{2 \times(\text { Precision } \times \text { Sensitivity }}{(\text { Precision }+ \text { Sensitivity })}\right) \times 100 \% \\
& \text { Specificity }=\left(\frac{T N}{F P+T N}\right) \times 100 \% \\
& \text { FNR }=\left(\frac{F N}{T P+F N}\right) \times 100 \% \\
& \text { FPR }=\left(\frac{F P}{F P+T N}\right) \times 100 \%
\end{aligned}
$$


Table 2 exhibits the performance evaluation matrices for the DenseNet201 model for class wise (anthracnose, gall machi, healthy leaf, powdery mildew, and red rust). We find from Table 2 that the accuracy is $98.00 \%$ for the identification of diseases of mango leaves. F1score, accuracy, sensitivity, specificity, FNR, and FPR have also been measured. The highest F1 score for powdery mildew, red rust, was 98.33\%. The highest precision, and sensivity found for anthracnose, powdery mildew, and red rust, is 98.33\%. The highest specificity, observed for anthracnose, gall machi, powdery mildew, red rust, is 99.58\%.The lowest FNR and FPR, found for anthracnose, powdery mildew, red rust, is $1.67 \%$ and $0.42 \%$ as well.

Table 3 exhibits the performance evaluation matrices for the InceptionResNetV2 model for class wise. We find from Table 3 that the accuracy is $96.67 \%$ for the identification of diseases of mango leaves using InceptionResNetV2 model. The highest F1 score and precision for red rust, was 98.33\%.The highest sensitivity, observed for gall machi, powdery mildew, and red rust is $98.33 \%$. The highest specificity, observed for anthracnose, and red rust is $99.58 \%$. The lowest FNR, found for powdery mildew, and red Rust is $1.67 \%$. For healthy leaf the lower FPR is $0.17 \%$.

Table 2. Class wise performance evaluation matrices for DenseNet201

\begin{tabular}{cccccccc}
\hline & & \multicolumn{7}{c}{ DenseNet201 } & & \\
Class & Accuracy & F1 & Precision & Sensitivity & Specificity & FNR & FPR \\
\hline Anthracnose & & $98.31 \%$ & $98.33 \%$ & $98.33 \%$ & $99.58 \%$ & $1.67 \%$ & $0.42 \%$ \\
Gall Machi & & $97.48 \%$ & $98.30 \%$ & $96.67 \%$ & $99.58 \%$ & $3.33 \%$ & $0.42 \%$ \\
Healthy Leaf & $98.00 \%$ & $97.52 \%$ & $96.72 \%$ & $98.33 \%$ & $99.17 \%$ & $1.67 \%$ & $0.83 \%$ \\
Powdery Mildew & & $98.33 \%$ & $98.33 \%$ & $98.33 \%$ & $99.58 \%$ & $1.67 \%$ & $0.42 \%$ \\
Red Rust & & $98.33 \%$ & $98.33 \%$ & $98.33 \%$ & $99.58 \%$ & $1.67 \%$ & $0.42 \%$ \\
\hline
\end{tabular}

Table 3. Class wise performance evaluation matrices for InceptionResNetV2

\begin{tabular}{|c|c|c|c|c|c|c|c|}
\hline \multicolumn{8}{|c|}{ InceptionResNetV2 } \\
\hline Class & Accuracy & $\mathrm{F} 1$ & precision & Sensitivity & Specificity & FNR & FPR \\
\hline Anthracnose & & $97.48 \%$ & $98.31 \%$ & $96.67 \%$ & $99.58 \%$ & $3.33 \%$ & $0.42 \%$ \\
\hline Gall Machi & & $94.92 \%$ & $96.55 \%$ & $93.33 \%$ & $99.17 \%$ & $6.67 \%$ & $0.83 \%$ \\
\hline Healthy Leaf & $96.67 \%$ & $95.08 \%$ & $93.55 \%$ & $96.67 \%$ & $98.33 \%$ & $3.33 \%$ & $0.17 \%$ \\
\hline Powdery Mildew & & $97.52 \%$ & $96.72 \%$ & $98.33 \%$ & $99.17 \%$ & $1.67 \%$ & $0.83 \%$ \\
\hline Red Rust & & $98.33 \%$ & $98.33 \%$ & $98.33 \%$ & $99.58 \%$ & $1.67 \%$ & $0.42 \%$ \\
\hline
\end{tabular}

Table 4 exhibits the performance evaluation matrices for the InceptionV3 model for class wise. We find from Table 4 that the accuracy is $96.67 \%$ for the identification of diseases of mango leaves using InceptionV3 model. F1score, accuracy, sensitivity, specificity, FNR, and FPR have also been measured. The highest F1 score for red rust was $98.33 \%$. The highest precision found for red rust is $98.33 \%$. The highest sensitivity observed for healthy leaf, powdery mildew, and red rust is $98.33 \%$. The highest specificity, observed for Anthracnose, and Red Rust is $99.58 \%$.The lowest FNR, found for healthy leaf, powdery mildew, and red rust, is $1.67 \%$. For anthracnose the lower FPR is $0.42 \%$.

Table 4. Class wise performance evaluation matrices for InceptionV3

\begin{tabular}{cccccccc}
\hline \multirow{2}{*}{ Class } & \multirow{2}{c}{ InceptionV3 } & & & \\
Accuracy & F1 & Precision & Sensitivity & Specificity & FNR & FPR \\
\hline Anthracnose & & $95.73 \%$ & $98.25 \%$ & $93.33 \%$ & $99.58 \%$ & $6.67 \%$ & $0.42 \%$ \\
Gall Machi & & $95.80 \%$ & $96.61 \%$ & $95.00 \%$ & $99.17 \%$ & $5.00 \%$ & $0.83 \%$ \\
Healthy Leaf & $96.67 \%$ & $96.72 \%$ & $95.16 \%$ & $98.33 \%$ & $98.75 \%$ & $1.67 \%$ & $1.35 \%$ \\
Powdery Mildew & & $96.72 \%$ & $95.16 \%$ & $98.33 \%$ & $98.75 \%$ & $1.67 \%$ & $1.25 \%$ \\
Red Rust & & $98.33 \%$ & $98.33 \%$ & $98.33 \%$ & $99.58 \%$ & $1.67 \%$ & $0.42 \%$ \\
\hline
\end{tabular}

Table 5 exhibits the performance evaluation matrices for the ResNet50 model for class wise. We find from Table 5 that the accuracy is $97.00 \%$ for the identification of diseases of mango leaves using RasNet50 model. F1score, accuracy, sensitivity, specificity, FNR, and FPR have also been measured. The highest F1 score and precesion for anthracnose, and red rust was 98.33\%. The highest sensitivity, observed for anthracnose, gall machi, healthy leaf, and red rust is $98.33 \%$. The highest specificity observed for anthracnose, gall machi, powdery mildew, and red rust is $99.58 \%$. The lowest FNR found for anthracnose, healthy leaf, and red rust is $1.67 \%$. For anthracnose, gall machi, powdery mildew, and red rust the lower FPR is $0.42 \%$. 
Table 5. Class wise performance evaluation matrices for ResNet50

\begin{tabular}{cccccccc}
\hline \multirow{2}{*}{ Class } & \multirow{7}{*}{ ResNet50 } & & \\
Accuracy & F1 & Precision & Sensitivity & Specificity & FNR & FPR \\
\hline Anthracnose & & $98.33 \%$ & $98.33 \%$ & $98.33 \%$ & $99.58 \%$ & $1.67 \%$ & $0.42 \%$ \\
Gall Machi & & $95.73 \%$ & $98.25 \%$ & $93.33 \%$ & $99.58 \%$ & $6.67 \%$ & $0.42 \%$ \\
Healthy Leaf & $97.00 \%$ & $95.16 \%$ & $92.19 \%$ & $98.33 \%$ & $97.92 \%$ & $1.67 \%$ & $2.08 \%$ \\
Powdery Mildew & & $97.48 \%$ & $98.31 \%$ & $96.67 \%$ & $99.58 \%$ & $3.33 \%$ & $0.42 \%$ \\
Red Rust & & $98.33 \%$ & $98.33 \%$ & $98.33 \%$ & $99.58 \%$ & $1.67 \%$ & $0.42 \%$ \\
\hline
\end{tabular}

Table 6 exhibits the performance evaluation matrices for the ResNet152V2 model for class wise. We find from Table 6 that the accuracy is $94.67 \%$ for the identification of diseases of mango leaves. F1score, accuracy, sensitivity, specificity, FNR, and FPR have also been measured. The highest F1 score for healthy leaf, was $95.87 \%$. The highest precision, found for red rust is $98.25 \%$. The highest sensitivity observed for powdery mildew, and red rust is $98.33 \%$. The highest specificity observed for red rust is $99.58 \%$. The lowest FNR found for powdery mildew, and red rust is $1.67 \%$. For red rust, the lower FPR is $0.42 \%$.

Table 7 exhibits the performance evaluation matrices for the Xception model for class wise. We find from Table 7 that the accuracy is $97.67 \%$ for the identification of diseases of mango leaves using Xception model. F1score, accuracy, sensitivity, specificity, FNR, and FPR have also been measured. The highest F1 score, precision and sensitivity for anthracnose, powdery mildew, and red rust was $98.33 \%$. The highest specificity, observed for anthracnose, gall machi, powdery mildew, and red rust is $99.58 \%$. The lowest FNR, found for anthracnose, healthy leaf, powdery mildew, and red rust is $1.67 \%$. For anthracnose, gall machi, powdery mildew, and red rust, the lower FPR is $0.42 \%$.

Table 6. Class wise performance evaluation matrices for ResNet152V2

\begin{tabular}{cccccccc}
\hline & & \multicolumn{7}{c}{ ResNet152V2 } \\
Class & Accuracy & F1 & Precision & Sensitivity & Specificity & FNR & FPR \\
\hline Anthracnose & & $95.80 \%$ & $96.61 \%$ & $95.00 \%$ & $99.17 \%$ & $5.00 \%$ & $0.83 \%$ \\
Gall Machi & & $93.10 \%$ & $96.43 \%$ & $90.00 \%$ & $99.17 \%$ & $10.00 \%$ & $0.83 \%$ \\
Healthy Leaf & $94.67 \%$ & $95.87 \%$ & $95.08 \%$ & $96.67 \%$ & $98.75 \%$ & $3.33 \%$ & $1.25 \%$ \\
Powdery Mildew & & $92.91 \%$ & $88.06 \%$ & $98.33 \%$ & $96.67 \%$ & $1.67 \%$ & $3.33 \%$ \\
Red Rust & & $95.73 \%$ & $98.25 \%$ & $93.33 \%$ & $99.58 \%$ & $6.67 \%$ & $0.42 \%$ \\
\hline
\end{tabular}

Table 7. Class wise performance evaluation matrices for Xception

\begin{tabular}{|c|c|c|c|c|c|c|c|}
\hline \multicolumn{8}{|c|}{ Xception } \\
\hline Class & Accuracy & $\mathrm{F} 1$ & Precision & Sensitivity & Specificity & FNR & FPR \\
\hline Anthracnose & & $98.33 \%$ & $98.33 \%$ & $98.33 \%$ & $99.58 \%$ & $1.67 \%$ & $0.42 \%$ \\
\hline Gall Machi & & $96.61 \%$ & $98.28 \%$ & $95.00 \%$ & $99.58 \%$ & $5.00 \%$ & $0.42 \%$ \\
\hline Healthy Leaf & $97.67 \%$ & $96.72 \%$ & $95.16 \%$ & $98.33 \%$ & $98.75 \%$ & $1.67 \%$ & $1.25 \%$ \\
\hline Powdery Mildew & & $98.33 \%$ & $98.33 \%$ & $98.33 \%$ & $99.58 \%$ & $1.67 \%$ & $0.42 \%$ \\
\hline Red Rust & & $98.33 \%$ & $98.33 \%$ & $98.33 \%$ & $99.58 \%$ & $1.67 \%$ & $0.42 \%$ \\
\hline
\end{tabular}

\section{CONCLUSION}

The discoveries of this research demonstrate that disease distinguishing proof from the image with the convolutional neural network is a solid strategy for high exactness automated distinguishing proof of mango disease. In this proposed work, six CNN models are utilized for five unique classes of mango disease. Image processing techniques have been used for the augmentation of the image data. The pre-trained transfer learning technique has been utilized here. After completion of training and testing the image data, a $5 \times 5$ confusion matrix has been generated. Based on the evaluation of seven performance metrics, the highest accuracy is $98.00 \%$ found for the DenseNet201 model. The main focus should be pointed toward settling few disadvantages in the proposed methodology also increase some more devising methods in this work. So in the future, we will try to focus on more diseases with this CNN technique to get better accuracy for those targeted diseases.

\section{REFERENCES}

[1] J. Bai, B. Wang, C. Chen, J. Chen, and Zhong-Hua Fu., "Inception-v3 Based Method of LifeCLEF 2019 Bird Recognition," In CLEF (Working Notes), 2019.

[2] P. Dhankhar, "ResNet-50 and VGG-16 for recognizing facial emotions," International Journal of Innovations in Engineering and Technology (IJIET), vol. 13, no. 4, pp. 126-130, 2019. 
[3] C. Lin, G. Zhao, Z. Yang, A. Yin, and X. Wang, "Cir-net: Automatic classification of human chromosome based on inception-resnet architecture," IEEE/ACM Transactions on Computational Biology and Bioinformatics, 2020, doi: 10.1109/TCBB.2020.3003445.

[4] C. Lin, L. Li, W. Luo, K. C. P. Wang, and J. Guo,"Transfer learning based traffic sign recognition using inceptionv3 model," Periodica Polytechnica Transportation Engineering, vol. 47, no. 3, pp. 242-250, 2019, doi: 10.3311/PPtr.11480.

[5] C. A. Ferreira, T. Melo, P. Sousa, M. Ines Meyer, and A. Campilho, "Classification of breast cancer histology images through transfer learning using a pre-trained inception resnet v2," In 2018 International Conference Image Analysis and Recognition, 2018, pp. 763-770, doi: 10.1007/978-3-319-93000-8_86.

[6] M. M. Rahman, et al., "Recognition of Local Birds of Bangladesh using MobileNet and Inception-v3," International Journal of Advanced Computer Science and Applications, vol. 11, no. 8, pp. 309-316, 2020, doi: 10.14569/IJACSA.2020.0110840.

[7] I. Z. Mukti, and D. Biswas, "Transfer learning based plant diseases detection using ResNet50." In 2019 4th International Conference on Electrical Information and Communication Technology (EICT), 2019, pp. 1-6, IEEE, doi: 10.1109/EICT48899.2019.9068805.

[8] I. Sharmin, N. F. Islam, I. Jahan, T. A. Joye, and Md. Riazur Rahman, "Machine vision based local fish recognition," SN Applied Sciences, vol. 1, no. 12, pp. 1-12, 2019, doi: 10.1007/s42452-019-1568-z.

[9] O. Prakash and A. Misra, "Important diseases of mango and their effect on production," Biol Memoirs, vol. 18, pp. 39-55, 1992.

[10] K. N. Shukla et al., "A review on image enhancement techniques," International Journal of Engineering and Applied Computer Science (IJEACS), vol. 2, no. 7, pp. 232-235, 2017.

[11] T. Lei, X. Jia, Y. Zhang, L. He, and H. Meng., "Significantly fast and robust fuzzy c-means clustering algorithm based on morphological reconstruction and membership filtering," IEEE Transactions on Fuzzy Systems, vol. 26, no. 5, pp. 3027-3041, 2018, doi: 10.1109/TFUZZ.2018.2796074.

[12] J. M. Patel and N. C. Gamit, "A review on feature extraction techniques in content based image retrieval," in International Conference on Wireless Communications, Signal Processing and Networking (WiSPNET), IEEE, 2016, pp. 2259-2263.

[13] R. M. Haralick, et al., "Textural features for image classification," IEEE Transactions on systems, man, and cybernetics, no. 6, pp. 610-621, 1973, doi: 10.1109/TSMC.1973.4309314.

[14] Y. Hu, Chun-xia Zhao, and Hong-nan Wang, "Directional analysis of texture images usinggray level co-occurrence matrix," in IEEE Pacific-Asia workshop on computational intelligence and industrial application, vol. 2, IEEE, pp. 277-281, 2008, doi: 10.1109/PACIIA.2008.279.

[15] D. L. Padmaja and B. Vishnuvardhan, "Comparative study of feature subset selection methods for dimensionality reduction on scientific data," in IEEE 6th International Conference on Advanced Computing (IACC), IEEE, 2016, pp. 31-34, doi: 10.1109/IACC.2016.16.

[16] V. Agarwal, "Research on data preprocessing and categorization technique for smartphone review analysis," International Journal of Computer Applications, vol. 131, no. 4, pp. 30-36, 2015, doi: 10.5120/ijca2015907309.

[17] A. Mikołajczyk, and M. Grochowski, "Data augmentation for improving deep learning in image classification problem," In 2018 international interdisciplinary PhD workshop (IIPhDW), pp. 117-122. IEEE, 2018, doi: 10.1109/IIPHDW.2018.8388338.

[18] S. Yu, C. Xu, and S. Jia, "Convolutional neural networks for hyperspectral image classification," Neurocomputing, vol. 219, pp. 88-98, 2017, doi: 10.1016/j.neucom.2016.09.010.

[19] A. A. Biswas, A. Majumder, A. Rajbongshi, and Md. M. Rahman, "Recognition of Local Birds using Different CNN Architectures with Transfer Learning," In 2021 International Conference on Computer Communication and Informatics (ICCCI), 2021, pp. 1-6, IEEE, doi: 10.1109/ICCCI50826.2021.9402686.

[20] M. Hossin, and M. N. Sulaiman, "A review on evaluation metrics for data classification evaluations," International Journal of Data Mining \& Knowledge Management Process, vol. 5, no. 2, 2015, doi: 10.5121/ijdkp.2015.5201.

[21] A. Majumder, Al A. Biswas, Md. M. Rahman, and A. Rajbongshi.,"Local Freshwater Fish Recognition Using Different CNN Architectures with Transfer Learning," International Journal on Advanced Science, Engineering and Information Technology, vol. 11, no. 3, pp. 1078-1083, 2021, doi: 10.18517/ijaseit.11.3.14134.

[22] N. Gupta, et al., "Accuracy sensitivity and specificity measurement of various classification techniques on healthcare data," IOSR Journal of Computer Engineering (IOSR-JCE), vol. 11, no. 5, pp. 70-73, 2013.

[23] C. Sammut, and Geoffrey I. "Webb, eds. Encyclopedia of machine learning," Springer Science \& Business Media, 2011.

[24] A. Rajbongshi, T. Sarker, Md. M. Ahamad, Md. And M. Rahman, "Rose Diseases Recognition using MobileNet," In 2020 4th International Symposium on Multidisciplinary Studies and Innovative Technologies (ISMSIT), pp. 1-7. IEEE, 2020, doi: 10.1109/ISMSIT50672.2020.9254420.

[25] K. M. Ghori, M. Imran, R. A. Abbasi, A. Ullah, and A. Nawaz, "Performance analysis of machine learning classifiers for non-technical loss detection," Journal of Ambient Intelligence and Humanized Computing, pp. 1-16, 2020, doi: 10.1007/s12652-019-01649-9. 